\title{
A Cadaveric Study on the Surface Projection of the Dorsal Scapular Nerve
}

Vuvi H. Nguyen' ${ }^{1}$ Hao (Howe) Liu ${ }^{2}$, Armando Rosales ${ }^{1}$, and Rustin Reeves ${ }^{{ }^{*}}$

${ }^{1}$ Center for Anatomical Sciences, University of North Texas Health Science Center, Fort Worth, TX 76107, USA

${ }^{2}$ Department of Physical Therapy, University of North Texas Health Science Center, Fort Worth, TX 76107, USA

\begin{abstract}
Background: Dorsal scapular nerve (DSN) syndrome is often associated by dull or aching pain along the medial border of the scapula that can radiate to the lateral aspect of the upper limb. The primary cause of this syndrome is due to the impingement or entrapment of this nerve at the middle scalene muscle. The purpose of this study is to identify the surface projection of the DSN relative to the middle scalene muscle by using the transverse plane of the laryngeal prominence and the posterior border of the sternocleidomastoid (SCM) muscle as reference points along with approximating the nerve's location using thumb interphalangeal joint (IPJ) width.
\end{abstract}

Methods: The surface location of the DSN was examined in 10 embalmed adult cadavers. The posterior border of the SCM muscle was palpated and outlined along with the transverse plane of the laryngeal prominence. A resin dye was injected at a distance of $2.08 \mathrm{~cm}(\sim 1$ thumb IPJ width) medial to the intersection of the posterior border of the SCM and the transverse plane of the laryngeal prominence. Dissections were performed to reveal and record the location of the dye. The distance between the location of the dye to the DSN was also measured.

Results: The overall accuracy of the injection study revealed that the scalene muscles were consistently located. Specifically, $50 \%$ of the injections were found at the middle scalene muscle, $20 \%$ was between the anterior and middle scalene muscles, $10 \%$ at the anterior scalene muscle, $10 \%$ between the middle and posterior scalene muscles, and $10 \%$ was located at the posterior scalene muscle.

Conclusion: This investigation will provide clinicians a useful and convenient method to determine the surface projection of the DSN at its entrapment site for the purpose of diagnosis and therapeutic treatment.

\section{Introduction}

The dorsal scapular nerve (DSN) is a motor nerve that primarily originates from the fifth cervical spinal nerve root in the brachial plexus [1-6]. Occasionally, in addition to C5, the DSN may also receive contributions from C4 [7-10]. The DSN arises within the posterior cervical triangle deep to the prevertebral fascia [11] and typically pierces the middle scalene muscle where it travels posteriorly between the posterior scalene and the serratus posterior superior muscles to provide motor innervation to the levator scapulae, rhomboid minor, and rhomboid major muscles. Collectively, all three of these muscles act to elevate and retract the scapula [12-17].

Several anatomical studies in the primary literature have indicated the variability of the DSN in terms of its spinal root origins and muscular innervations. For example, Shilal et al. (2015) reported that the DSN not only receive contributions from C5 and C6 but also communicated with branches from the long thoracic nerve [18]. Similarly, Ballestero's and Ramirez's study reported that nearly $48 \%$ of the DSNs branched from C5 while nearly $30 \%$ shared a trunk with the long thoracic nerve [19]. A recent cadaveric study by Nguyen et al. (2016) found that approximately $70 \%$ of the DSN originated from C5 while $22 \%$ arose from C4 and 8\% branched from C6 [13]. Chen et al. (1995) also reported that in addition to C5, the DSN received variable contributions throughout C4-T1 [20]. In addition, there are varying reports regarding the muscular innervations of the DSN. For example, a case study in Japan reported that the DSN innervated the serratus posterior superior muscle [21]. In a study by Frank et al. [22], they reported that the DSN innervated the levator scapulae muscle in only 11 out of 35 neck specimens. Similarly, Nguyen et al.'s study also found that $48 \%$ of the DSN supplied the levator scapulae muscle only whereas $52 \%$ of the nerve supplied the levator scapulae as well as the rhomboid major and minor muscles.

DSN syndrome is characterized by general symptoms of sharp, dull, or aching pain along the medial border of the scapula that radiates to the lateral surface of the arm and forearm [23]. Patients also report dysfunction of their shoulders as well as pain in their neck and back region [20]. DSN syndrome is often caused by the entrapment or impingement of this nerve at the middle scalene muscle, because the DSN often pierces this muscle [13,24-26]. However, because the DSN lacks sensory branches, the entrapment of this nerve is often overlooked during clinical diagnosis of back and interscapular pain $[17,24]$. In addition, the variability in the anatomy of the DSN in terms of its spinal root origins and muscular innervations may also be another factor in which DSN impingement is frequently missed [13]. Occupations that require overhead work, such as painters and electricians, make these particular individuals more susceptible to DSN impingement [17]. There are also documented injuries of the DSN amongst athletes such as volleyball and basketball players, judo, and body builders [24, 27-29]. For example, along with injury to the

*Corresponding Author: Dr. Rustin Reeves, Center for Anatomical Sciences, The University of North Texas Health Science Center at Fort Worth 3500 Camp Bowie Blvd, Fort Worth, TX 76107, USA, Tel: 817-735-2050; E-mail: rustin.reeves@unthsc.edu

Citation: Nguyen VH, Liu H, Rosales A, Reeves R (2017) A Cadaveric Study on the Surface Projection of the Dorsal Scapular Nerve. Int J Phys Ther Rehab 3: 131. doi: https://doi.org/10.15344/2455-7498/2017/131

Copyright: (c) 2017 Nguyen et al. This is an open-access article distributed under the terms of the Creative Commons Attribution License, which permits unrestricted use, distribution, and reproduction in any medium, provided the original author and source are credited. 
suprascapular nerve, the DSN was also injured in two sibling volleyball players. Both siblings reported pain in their right shoulders and scapular region as well as mild winging of their right scapulas with weakness of the rhomboid muscles [30]. There are also case reports in which a lesion to or neuropathy of the DSN caused scapular winging [31-33]. For example, Akgun et al. [17] reported a 51-year-old man who damaged his DSN after lifting a heavy box overhead. As a result from this lesion, he developed right shoulder pain as well as weakness of arm abduction and winging of his right scapula.

Current treatments to help resolve patients of their pain from DSN syndrome include muscle manipulation at the scalene muscles and/ or nerve block injection $[13,20,34]$. According to Walther, soft tissue manipulation can be performed by passively extending the patient's neck in order to specifically stretch their middle scalene muscle of the affected side [35]. Another form of conservative treatment is directly anesthetizing the DSN. In this method, a nerve block injection that is typically guided via ultrasound, is administered in order to relieve patients of their symptoms [16,25,36,37]. Although rare, surgical intervention such as lesion of the middle scalene muscle have also been reported to relieve patients from their pain [20]. In both types of these conservative and surgical treatments, it is imperative for rehabilitation professionals to be aware of other important anatomical structures surrounding the scalene muscles of the neck such as the phrenic nerve as well as the roots and trunks of the brachial plexus in order to reduce the risk of injuring these structures.

Our previous study of the DSN investigated the relationship of this nerve as it crosses the middle scalene muscle relative to the transverse plane of the laryngeal prominence [13]. Average distances from the transverse plane of the laryngeal prominence to where the DSN entered, crossed, and exited the middle scalene muscle were reported. We used data from our previous study, then added to those anatomical data by presenting thumb interphalangeal joint (IPJ) width to approximate and predict the surface projection of the DSN. This was done relative to its site of entrapment (the middle scalene muscle) while using the transverse plane of the laryngeal prominence and the posterior border of the SCM muscle as anatomical landmarks. According to Liu et al. [38], thumb width is a convenient measurement tool commonly used by clinicians such as physical therapists to measure the distance from the location of pain to a given body landmark. Injection studies were performed to test the accuracy of using thumb IPJ width to locate the site of DSN entrapment at the middle scalene muscle.

The overall purpose of this study is to provide a convenient method for rehabilitation professionals to examine, diagnose, and treat patients with possible DSN impingement through the use of thumb IPJ width while using the transverse plane of the laryngeal prominence and the posterior border of the SCM as reference points. This method will assist clinicians in evaluating and implementing appropriate therapeutic treatments to patients who may exhibit symptoms of DSN syndrome.

\section{Materials \& Methods}

The surface projection of the dorsal scapular nerve was examined in 10 embalmed adult cadavers (6 males and 4 females) obtained through the Willed Body Program, Center for Anatomical Sciences, at the University of North Texas Health Science Center (UNTHSC) in Fort Worth, Texas. The age of the donors span from 68 to 92 years with a mean age of 80 years. The self-reported ethnicities of the donors are Caucasian. The cadavers are individually wrapped in cotton shroudswithMarylandStateWettingagent(HydrolChemicalCompany,
Yeadon, PA.) and are stored in metal tanks located in the UNTHSC Gross Anatomy Laboratory.

The cadavers used in this study have not been previously dissected and therefore, all skin in the neck region remained intact. The posterior border of the sternocleidomastoid (SCM) muscle was first identified and palpated. A transverse plane through the laryngeal prominence was established using a $90^{\circ}$-angled ruler. A grease pencil was used to outline the posterior border of the SCM muscle as well as mark the transverse plane of the laryngeal prominence to create reference points. An injection was made at approximately $2.08 \mathrm{~cm}$ medial from the intersection of the posterior border of the SCM and the transverse plane of the laryngeal prominence (Figure 1(a)). This value is the average distance at which the DSN exited the middle scalene muscle from the transverse plane of the laryngeal prominence as reported from our previous research [13]. In addition, $2.08 \mathrm{~cm}$ is equivalent to approximately one thumb IPJ width as reported from Liu et al.'s study in which average thumb IPJ width is approximately $2.0 \pm 0.4 \mathrm{~cm}$ [38]. For injection, a polyurethane resin (PU4ii) with a proprietary blue dye was prepared following the manufacturer's instructions (vasQtec, Zürich, Switzerland). Approximately $0.1 \mathrm{ml}$ of the resin dye was injected at a depth of $1 \mathrm{~cm}$ using a $1 \mathrm{ml}$ syringe with a 22 gauge needle. The polyurethane resin was allowed to solidify for 24 hours post-injection. Dissections were then made along the posterior border of the SCM to reveal the location of the injection site as indicated by the blue dye. The distance of the dye to the DSN was measured using an electronic sliding caliper (Carrera Precision Corp.). All injections and dissections were performed on the left side of the neck region. On the right side of the neck, a previous incision was made to access vasculature for the embalming of our cadavers. Therefore, important structures such as the scalene muscles and the DSN were often damaged on that side. Dissection images were taken with a digital camera (Nikon Coolpix S6200).

\section{Results}

The surface projection of the DSN was investigated in 10 embalmed adult cadavers. Measurements were also taken between the site of injection and the actual location of the DSN at the midpoint of the middle scalene muscle. The results of the injection study revealed that in 5 cadavers, the resin dye was located directly at the middle scalene muscle as the DSN either pierces or crosses anteriorly to this muscle. On one cadaver, the dye was located at the anterior scalene muscle and the distance between the location of the dye to the DSN was 0.683 $\mathrm{cm}$. On two cadavers, the dye was located between the anterior and middle scalene muscles. The average distances between the location of the dye and the DSN at these injections was approximately 1.40 $\mathrm{cm}$. In another cadaver, the dye was between the middle and posterior scalene muscles and the distance between the location of this injection to the DSN was about $0.676 \mathrm{~cm}$. On the last cadaver, the dye was found at the posterior scalene muscle and the distance between this injection site and the DSN was $0.832 \mathrm{~cm}$. Figure 1 is an example of our injection study showing the blue dye at the middle scalene muscle and the DSN crosses anteriorly to this muscle. It was also observed that in relation to the middle scalene muscle, $50 \%$ of the DSN pierced this muscle whereas $40 \%$ of the DSN crossed anterior to the middle scalene muscle and $10 \%$ of the nerve traveled posterior to the middle scalene muscle.

\section{Discussion}

We used previous data from Nguyen et al. [13] in order to estimate the surface projection of the DSN relative to the middle scalene muscle. 
Citation: Nguyen VH, Liu H, Rosales A, Reeves R (2017) A Cadaveric Study on the Surface Projection of the Dorsal Scapular Nerve. Int J Phys Ther Rehab 3: 131.

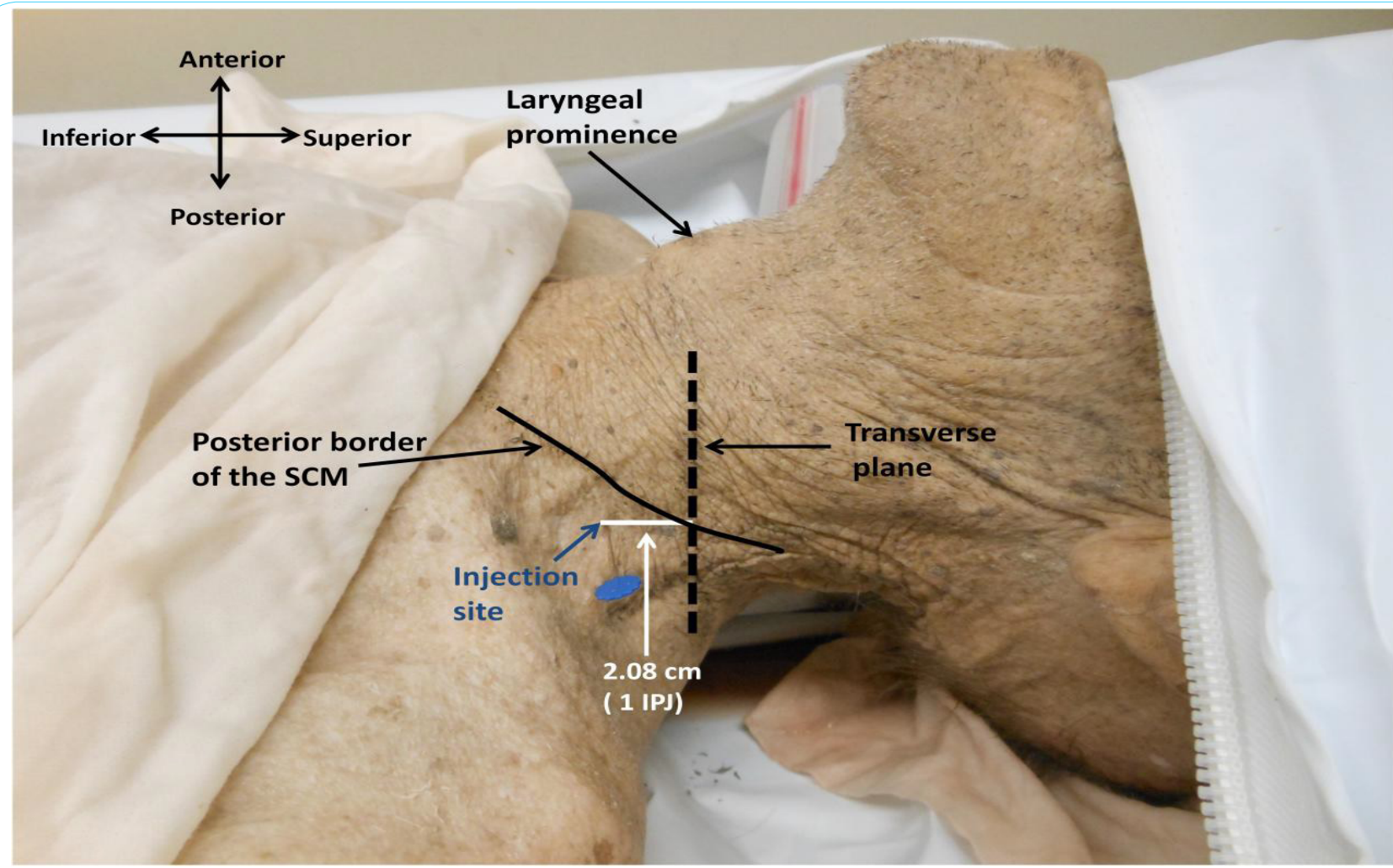

Figure 1a: The surface anatomy of the left neck region of an 81-year-old male cadaver in the supine position. The black dashed lines represents the transverse plane of the laryngeal prominence. The black solid line indicates the posterior border of the sternocleidomastoid (SCM) muscle. The white solid line represents the distance of $2.08 \mathrm{~cm}(\sim 1$ thumb IPJ width) at which the injection was performed. The blue pin indicates the injection site.

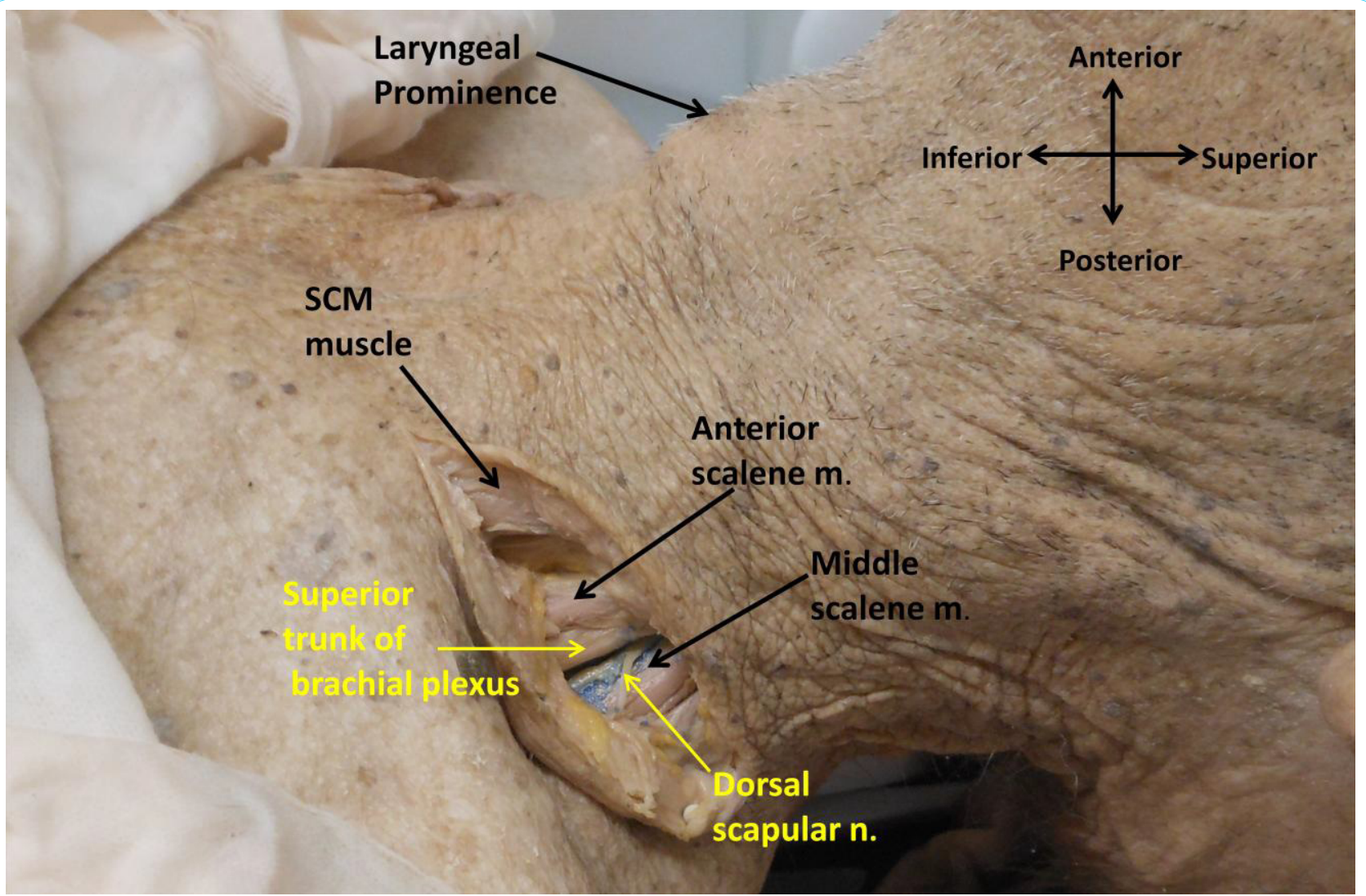

Figure $1 \mathrm{~b}$. The dissection revealed the location of the injection site as indicated by the blue stain on the middle scalene muscle as the DSN crosses anterior to this muscle. 
Citation: Nguyen VH, Liu H, Rosales A, Reeves R (2017) A Cadaveric Study on the Surface Projection of the Dorsal Scapular Nerve. Int J Phys Ther Rehab 3: 131. doi: https://doi.org/10.15344/2455-7498/2017/131

Page 4 of 5

The average distance, $2.08 \mathrm{~cm}( \pm 0.96 \mathrm{~cm})$, was chosen from our previous research as the distance for the injection site from the intersection of the transverse plane of the laryngeal prominence and the posterior border of the SCM muscle. This distance for the injection site was chosen for several reasons. Because $2.08 \mathrm{~cm}$ is the measurement at which the DSN exited the middle scalene muscle, this value is located at the most lateral border of this muscle. Therefore, important anatomical structures such as the phrenic nerve and the superior trunk of the brachial plexus would be farthest away from the injection site. This information is especially important for rehabilitation professionals in order to avoid injuring these anatomical structures during a nerve block injection. In addition, for therapists and clinicians, $2.08 \mathrm{~cm}$ is approximately 1 thumb IPJ width which makes this measurement clinically useful in pinpointing the surface projection of the DSN while using the reference points of the posterior border of the SCM muscle and the transverse plane of the laryngeal prominence.

The results of our investigation revealed that the surface location of the anterior, middle, and posterior scalene muscles were consistently identified when approximating the surface projection of the DSN using 1 thumb IPJ width medial to the intersection of the posterior border of the SCM muscle and transverse plane of the laryngeal prominence. Although we accurately identified the surface location of the DSN at its typical entrapment site (the middle scalene muscle) in $50 \%$ of the injections performed, the distances between the dye at other sites within the scalene muscles to the actual location of the DSN were measured. In those measurements, the average distance between the injected dye and the DSN was less than $1.0 \mathrm{~cm}$ which is less than half the distance of 1 thumb IJP width. Clinically, rehabilitation professionals could use these measurements as a radius to approximate the area of a circle at or very near to the DSN's position at the middle scalene muscle. This would allow professionals to treat patients with DSN syndrome by performing circular tissue manipulations within the surface projection of the middle scalene muscle.

\section{Conclusion}

Because the surface projection of the DSN has not been previously reported, the overall significance of this research is to provide easily identifiable reference points for clinicians to locate the nerve. Utilizing the posterior border of the SCM muscle as well as the transverse plane of the laryngeal prominence, clinicians' ability to accurately and efficiently locate the site of DSN entrapment will improve. In addition, using these reference points combined with a simple 1 thumb IPJ width measurement, this method may prove to be very useful for rehabilitation professionals to examine, diagnose, and conservatively treat patients with DSN impingement. Future studies in investigating the effectiveness of our method in a patient population along with locating the DSN via ultrasound could be beneficial in validating our proposed method.

\section{Competing Interests}

The authors declare that they have no competing interests.

\section{Author Contributions}

Vuvi Nguyen: contributed to the concept and design, data acquisition, data analysis and interpretation, and writing of manuscript.

Hao (Howe) Liu: contributed to the concept and design, data interpretation, and manuscript editing

Armando Rosales: contributed to data interpretation and manuscript editing
Rustin Reeves: contributed to the concept and design, data analysis and interpretation, and manuscript editing for final approval

\section{Acknowledgements}

The authors thank the selfless gifts made by body donors to the Willed Body Program, Center for Anatomical Sciences at the University of North Texas Health Science Center in Fort Worth. This research would not be possible without their generosity.

\section{References}

1. Moses KP (2013) Atlas of clinical gross anatomy, 2nd edn, Philadelphia PA: Elsevier/Saunders. xvii, $633 p$

2. Slaby F, McCune SK, Summers RW (1994) Gross anatomy in the practice of medicine, Philadelphia: Lea \& Febiger. xxv, 699 p.

3. Rogers AW, Jacobs S (1992) Textbook of anatomy, Edinburgh ; New York: Churchill Livingstone. xi, $779 \mathrm{p}$.

4. Snell RS (2004) Clinical anatomy, 7th edn., Philadelphia: Lippincott Williams \& Wilkins. $x, 1012 p$.

5. Drake RL (2015) Gray's anatomy for students. 3rd edn., Philadelphia, PA Churchill Livingstone/Elsevier. xxv, 1161 pages.

6. Hartwig WC (2008) Fundamental anatomy. 2008, Philadelphia: Wolters Kluwer Health/Lippincott Williams \& Wilkins. ix, $417 \mathrm{p}$.

7. Moore KL, Dalley AF, Agur AMR (2014) Clinically oriented anatomy, 7th edn., Philadelphia: Wolters Kluwer/Lippincott Williams \& Wilkins Health. xxviii, $1134 \mathrm{p}$

8. Hansen JT, Netter FH (2010) Netter's clinical anatomy, 2nd edn., Philadelphia: Saunders/Elsevier. xviii, 470 p.

9. Gilroy AM (2016) Atlas of anatomy. 3rd edn., New York: Thieme. p.

10. Netter FH (2014) Atlas of human anatomy. 6th edn,, Philadelphia, PA: Saunders/Elsevier.

11. Trescot AM (2016) Dorsal Scapular Nerve Entrapment, in Peripheral Nerve Entrapments: Clinical Diagnosis and Management, M.D.A.F.M.A. Trescot, Springer International Publishing: Cham. p. 315-324.

12. Tubbs RS, Tyler-Kabara EC, Aikens AC, Martin JP, Weed LL, et al. (2005) Surgical anatomy of the dorsal scapular nerve. J Neurosurg 102: 910-911.

13. Nguyen VH, Liu HH, Rosales A, Reeves R (2016) A Cadaveric Investigation of the Dorsal Scapular Nerve. Anat Res Int 2016: 4106981.

14. Clemente CD (1987) Anatomy, a regional atlas of the human body, 3rd ed., Baltimore: Urban \& Schwarzenberg. 439 p.

15. Larsen WJ (2002) Anatomy : development, function, clinical correlations, Philadelphia: Saunders. $x, 741 \mathrm{p}$.

16. Auyong DB, Cabbabe AA2 (2014) Selective blockade of the dorsal scapular nerve for scapula surgery. J Clin Anesth 26: 684-687.

17. Akgun K, Aktas I, Terzi Y (2008) Winged scapula caused by a dorsal scapular nerve lesion: a case report. Arch Phys Med Rehabil 89: $2017-$ 2020.

18. Shilal P, Sarda RK, Chhetri K, Lama P, Tamang BK (2015) Aberrant Dual Origin of the Dorsal Scapular Nerve and Its Communication with Long Thoracic Nerve: An Unusual Variation of the Brachial Plexus. J Clin Diagn Res 9: AD01-AD02.

19. Ballesteros LE, Ramirez LM (2007) Variations of the origin of collateral branches emerging from the posterior aspect of the brachial plexus. $J$ Brachial Plex Peripher Nerve Inj 2: 14

20. Chen D, Gu Y, Lao J, Chen L (1995) Dorsal scapular nerve compression. Atypical thoracic outlet syndrome. Chin Med J (Engl) 108: 582-585.

21. Kida MY, Tani M (1993) The human superior posterior serratus muscle supplied by both the intercostal and dorsal scapular nerves. Kaibogaku Zasshi 68: 162-168. 
Citation: Nguyen VH, Liu H, Rosales A, Reeves R (2017) A Cadaveric Study on the Surface Projection of the Dorsal Scapular Nerve. Int J Phys Ther Rehab 3: 131. doi: https://doi.org/10.15344/2455-7498/2017/131

Page 5 of 5

22. Frank DK, Wenk E, Stern JC, Gottlieb RD, Moscatello AL (1997)A cadaveric study of the motor nerves to the levator scapulae muscle. Otolaryngol Head Neck Surg 117: 671-680.

23. Saporito A(2013) Dorsal scapular nerve injury: a complication of ultrasoundguided interscalene block. Br J Anaesth 111: 840-841.

24. Sultan HE, Younis El-Tantawi GA (2013) Role of dorsal scapular nerve entrapment in unilateral interscapular pain. Arch Phys Med Rehabil 94 $1118-1125$

25. Kim YD, Yu JY, Shim J, Heo HJ, Kim H (2016) Risk of Encountering Dorsal Scapular and Long Thoracic Nerves during Ultrasound-guided Interscalene Brachial Plexus Block with Nerve Stimulator. Korean J Pain 29: 179-84.

26. Jenkins GW, Tortora GJ (2013) Anatomy and physiology : from science to life. 3rd ed, Hoboken, NJ: Wiley. xliii, 987, 2, 2, 2, 13, 31, 3, 31 p.

27. Akuthota V, Herring SA (2009) Nerve and vascular injuries in sports medicine, New York; London: Springer. xiv, 204 p.

28. Jerosch J, Castro WH, Geske, Damage of the long thoracic and dorsal scapular nerve after traumatic shoulder dislocation: case report and review of the literature. Acta Orthop Belg 56: 625-627.

29. Mondelli M, Cioni R, Federico A (1998) Rare mononeuropathies of the upper limb in bodybuilders. Muscle Nerve 21: 809-812.

30. Ravindran M (2003) Two cases of suprascapular neuropathy in a family. $\mathrm{Br}$ J Sports Med 37: 539-541.

31. Kuhn JE, Plancher KD, Hawkins RJ (1995) Scapular Winging. J Am Acad Orthop Surg 3: 319-325.

32. Duralde XA (1995) Evaluation and treatment of the winged scapula. J South Orthop Assoc 4: 38-52.

33. Argyriou AA, Karanasios P, Makridou A, Makris N (2015) Dorsal scapular neuropathy causing rhomboids palsy and scapular winging. J Back Musculoskelet Rehabil 28: 883-885.

34. Walther DS (1988) Applied kinesiology : synopsis, Pueblo, Colo.: Systems DC. xii, $572 \mathrm{p}$.

35. Walther DS (2000) Applied kinesiology : synopsis. 2nd ed. Pueblo, Colo. Systems DC. xii, $627 \mathrm{p}$.

36. Burckett-St Laurent D, Chan V, Chin KJ (2014) Refining the ultrasoundguided interscalene brachial plexus block: the superior trunk approach. Can J Anaesth 61: 1098-1102.

37. Hanson NA, Auyong DB (2013) Systematic ultrasound identification of the dorsal scapular and long thoracic nerves during interscalene block. Reg Anesth Pain Med 38: 54-57.

38. Liu H, Fletcher J, Dailey M, Persson M (2009) Surface projection of the radial tunnel under the supinator muscle: A cadaver study. Advances in Physiotherapy 11: 234-241. 